Pacific Journal of Mathematics

INTERPOLATION IN STRONGLY LOGMODULAR ALGEBRAS 


\title{
INTERPOLATION IN STRONGLY LOGMODULAR ALGEBRAS
}

\section{RAHMAN YOUNIS}

\begin{abstract}
Let $A$ be a strongly logmodular subalgebra of $C(X)$, where $X$ is a totally disconnected compact Hausdorff space. For $s$ a weak peak set for $\mathbf{A}$, define $A_{S}=\left\{f \in C(X):\left.\left.f\right|_{s} \in A\right|_{S}\right\}$. We prove the following:
\end{abstract}

THEOREM 1. Let $s$ be a weak peak set for $A$. If $b$ is an inner function such that $\left.b\right|_{S}$ is invertible in $\left.A\right|_{S}$ then there exists a function $F$ in $A \cap C(X)^{-1}$ such that $F=\bar{b}$ on $s$.

Theorem 2. Let $s$ be a weak peak set for $A$. If $U \in C(X)$, $|U|=1$ on $s$ and $\operatorname{dist}\left(U, A_{S}\right)<1$, then there exists a unimodular function $\tilde{U}$ in $C(X)$ such that $\tilde{U}=U$ on $s$ and $\operatorname{dist}(\tilde{U}, A)<1$.

1. Introduction. The purpose of this paper is to prove certain properties related to strongly logmodular algebras.

In their study of Local Toeplitz operators, Clancey and Gosselin [3] established one of these properties in a special case $\left(H^{\infty}\right)$ under a highly restrictive condition. In [7], the author proved this property for $H^{\infty}$ without any condition.

In the present paper, we obtain this and another property for arbitrary strongly logmodular algebras. The proofs in [3] and [7] use special properties of $H^{\infty}$ that are not shared by arbitrary strongly logmodular algebra. In the present work we use new techniques.

Let $A$ be a strongly logmodular subalgebra of $C(X)$, where $X$ is a totally disconnected compact Hausdorff space. If $s$ is a weak peak set for $A$, define $A_{s}=\left\{f \in C(X):\left.\left.f\right|_{s} \in A\right|_{s}\right\}$. The main results of this work are: Theorem 3.2. Let $s$ be a weak peak set for $A$, and let $b$ be an inner function such that $\left.b\right|_{s}$ is invertible in $\left.A\right|_{s}$. Then there exists a function $F$ in $A \cap C(X)^{-1}$ such that $F=\bar{b}$ on $s$.

TheOREm 3.1. Let $s$ be a weak peak set for $A$, and let $u$ be in $C(X)$ such that $|u|=1$ on $s$ and $\operatorname{dist}\left(u, A_{s}\right)<1$. There exists $a$ unimodular function $\tilde{u}$ in $C(X)$ such that $\tilde{u}=u$ on $s$ and $\operatorname{dist}(\widetilde{u}, A)<1$.

2. Preliminaries. Let $X$ be a compact Hausdorff space. We denote by $C(X)\left[C_{R}(X)\right]$ the space of continuous complex [real] valued functions on $X$. The algebra $C(X)$ is a Banach algebra under the supremum norm $\|f\|_{\infty}=\sup \{|f(x)|: x \in X\}$.

Let $A$ be a function subalgebra of $C(X)$. A subset $S$ of $X$ is 
said to be a peak set for $A$ if there exists $f$ in $A$ such that $f=1$ on $S$ and $|f|<1$ off $S$. A set $S$ is a weak peak set for $A$ if $S$ is an arbitrary intersection of peak sets for $A$. Let $A^{-1}$ denote the group of invertible elements in $A$ and $\log \left|A^{-1}\right|=\left\{\log |f|: f \in A^{-1}\right\}$.

A function algebra $A$ is called a strongly logmodular subalgbra of $C(X)$ if $\log \left|A^{-1}\right|$ is equal to $C_{R}(X)$. The reader is referred to [2] and [4] for many of the basic properties of weak peak sets and additional properties of function algebra and to [5] and [1] for discussions concerning strongly logmodular algebras.

Let $A$ denote a fixed closed subalgebra of $C(X)$ which contains the constants. Let $B$ be a closed subalgebra of $C(X)$ which contains $A$. We define $B_{1}$ to be the closed subalgebra of $C(X)$ generated by $A$ and $\left\{f^{-1}: f \in A \cap B^{-1}\right\}$. It is clear that $A \subset B_{1} \subset B \subset C(X)$. If $B=B_{1}$, then $B$ is called a Douglas algebra.

A function $b$ in $A$ is called an inner function if $|b|=1$. For a strongly logmodular algebra $A$ on $X$, there is a useful characterization of $B_{1}$ in $[1, \mathrm{p} .8]$, which says that $B_{1}$ is the closed subalgebra generated by $A$ and $\{\bar{b} \in B: b$ is an inner function $\}$.

3. The main result. Throughout this section, $A$ will denote a fixed strongly logmodular algebra on $X$, where $X$ is a compact, totally disconnected Hausdorff space. Examples of such algebras can be found in [5] and [6].

Let $s$ be a subset of $X$ which is a weak peak set for $A$. Define $A_{S}=\left\{f \in C(X):\left.\left.f\right|_{s} \in A\right|_{s}\right\}$. The algebra $A_{S}$ is closed in $C(X)$ since $\left.A\right|_{s}$ is closed in $\left.C(X)\right|_{s}$. For $u$ in $C(X)$, we define $\operatorname{dist}_{S}(u, A)=$ $\inf \left\{\|u-h\|_{s}: h \in A\right\}$ and $\operatorname{dist}\left(u, A_{s}\right)=\inf \left\{\|u-h\|_{\infty}: h \in A_{s}\right\}$, where $\|u-h\|_{S}=\sup \{|u(x)-h(x)|: x \in S\}$. It is not difficult to see that $\operatorname{dist}\left(u, A_{s}\right)=\operatorname{dist}_{S}(u, A)$ for any $u$ in $C(X)$.

Our main result is as follows:

THEOREM 3.1. Let $s$ be a weak peak set for $A$, and let $u$ be in $C(X)$ such that $|u|=1$ on $s$ and $\operatorname{dist}\left(u, A_{s}\right)<1$. Then there exists a unimodular function $\tilde{u}$ in $C(X)$ such that $\tilde{u}=u$ on $s$ and $\operatorname{dist}(\widetilde{u}, A)<1$.

In the special case of $A=H^{\infty}$ (the Hardy space of the unit circle) the above theorem appeared in [7] which answers a question raised in [3].

To prove Theorem 3.1, we need the following special case of [1, Theorem 4.1].

TheOREM A. Let $A$ be a strongly logmodular subalgebra of $C(X)$ 
and $J$ be an ideal in $C(X)$, where $X$ is a totally disconnected compact Hausdorff space. Then the closure of $A+J$ is a Douglas algebra.

Theorem 3.1 follows from the following fact, which is interesting in its own right.

THEOREM 3.2. Let $s$ be a weak peak set for $A$, and let $b$ be an inner function such that $\left.b\right|_{S}$ is invertible in $\left.A\right|_{s}$. Then there exists a function $F$ in $A \cap C(X)^{-1}$ such that $F=\bar{b}$ on $s$.

Proof. Step 1. There is a peak set $E \supset s$ such that $\left.b\right|_{E} \in A_{E}^{-1}$. If not, there is a $\dot{\phi}_{E} \in M\left(A_{E}\right)$ such that $\dot{\phi}_{E}(b)=0$. Since $M\left(A_{E}\right) \subset M(A)$, which is compact we can choose a convergent subnet $\phi_{E}^{\prime} \rightarrow \dot{\phi}$. Clearly $\phi \in M\left(A_{S}\right)$, and $\phi(b)=0$ by continuity, contradicting $\left.b\right|_{S} \in A_{S}^{-1}$.

Step 2. Let $h$ peaks on $s$. Let $\phi \in M(A), \phi(h)=1$, and $\mu$ be the positive measure representing $\phi$ and supp $\mu$ be its support. Since $|h| \leqq 1$ and $\phi(h)=\int h d \mu=1$, we have $h=1$ on supp $\mu$. Because $h=1$ exactly on $s$, we have $\operatorname{supp} \mu \subset s$. This shows that $\phi \in M\left(A_{s}\right)$. Since $\left.b\right|_{s} \in A_{s}^{-1}, \phi(b) \neq 0$. Thus $1-h$ and $b$ have no common zeros on $M(A)$, and thus by [2, p. 27], there are $f, g \in A$ with $f b+$ $g(1-h)=1$.

Step 3. Fix $c>2\|g\|_{\infty}$, where $g$ is as in step (2). Let $E=$ $\{x \in X:|1-h|<1 / 6 c\}$. There exists a clopen set $W$ such that $s \subset W \subset E$. On the set $X \backslash W$ we have $|1-h|>\delta$, for some positive number $\delta$. Let $g_{1}=(c / 2) \chi_{W}+(11 / 6+c)(1 / \delta) \chi_{X \backslash W}$. Certainly, $g_{1} \in C(X)^{-1}$. Since $A$ is strongly logmodular, there exists $G \in A^{-1}$ such that $\log \left|g_{1}\right|=\log |G|$. Thus $|G|=c / 2$ on $W$ and $|G|=(11 / 6+c)(1 / \delta)$ on $X \backslash W$.

From the identity $f b+g(1-h)=1$, we have the following inequalities. On $W:|f|=|1-g(1-h)| \geqq 1-|g||1-h| \geqq 1-c / 2 \cdot 1 / 6 c=$ $1-\mathrm{T} / 12=11 / 12$, and on $X:|f| \leqq 1+|g||1-h| \leqq 1+c / 2 \cdot 2=1+c$. Let $F=f-G(1-h)$. Certainly, $F$ is in $A$ and $F=f=\bar{b}$ on s. Hence on $W$ we have that

$$
\begin{aligned}
|F| & \geqq|f|-|G||1-h| \\
& \geqq 11 / 12-c / 2 \cdot 1 / 6 c=5 / 6
\end{aligned}
$$

and

$$
\begin{aligned}
|F| & \geqq|G||1-h|-|f| \\
& \geqq(11 / 6+c)(1 / \delta) \cdot \delta-(1+c) \\
& =11 / 6+c-1-c=5 / 6 \quad \text { on } \quad X \backslash W .
\end{aligned}
$$


Thus $F \in A \cap C(X)^{-1}$. This ends the proof of the theorem.

Proof of Theorem 3.1. Without loss of generality we can assume that $|u|=1$ on $X$. It is easy to see that $A_{s}=A+J$, where $J=$ $\{f \in C(X): f(s)=0\}$. Thus, by Theorem $\mathrm{A}$, we have that $A_{S}$ is a Douglas algebra. From the inequality, $\operatorname{dist}\left(u, A_{S}\right)<1$, we have $\|u-g \bar{b}\|_{\infty}<1$, for some $g$ in $A$ and some inner function $b$ which is invertible in $A_{s}$. Consequently, $\operatorname{Re} \bar{u} \bar{b} g \geqq \delta_{1}>0$, for some positive number $\delta_{1}(\operatorname{Re} f$ denotes the real part of $f)$. By Theorem 3.2, there exists $F$ in $A \cap C(X)^{-1}$ such that $F=\bar{b}$ on s. Since $|F| \geqq \delta_{2}>0$, for some positive number $\delta_{2}$, we have $\operatorname{Re} \bar{u} \bar{b} \bar{F} /|F| F g=|F| \operatorname{Re} \bar{u} \bar{b} g \geqq$ $\delta_{1} \delta_{2}>0$. Thus there exists a positive real number $R>0$ such that $\|R-\bar{u} \bar{b} \bar{F} /|F| F g\|_{\infty}<R$. Hence $\|1-\bar{u} \bar{b} \bar{F} /|F| F g / R\|_{\infty}<1$. Set $\widetilde{u}=$ $u b F /|F|$; then $|\widetilde{u}|=1, \tilde{u}=u$ on $s$, and the last inequality shows that dist $(\tilde{u}, A)<1$. This ends the proof of the theorem.

The following corollary is a generalization of Theorem 3.2.

Corollary 3.3. If $s$ is a weak peak set for $A$ and $f$ in $C(X)$ such that $\left.f\right|_{S}$ is invertible in $\left.A\right|_{s}$, then there exists $G$ in $A \cap C(X)^{-1}$ such that $G=f$ on $s$.

Proof. The hypothesis that $\left.f\right|_{s}$ is invertible in $\left.A\right|_{s}$ shows that $f(x) \neq 0$ for all $x \in s$. Let $W$ be a clopen set of $X$ such that $f(x) \neq 0$ for all $x$ in $W$. The function $f \chi_{W}+1-\chi_{W} \in C(X)^{-1}$, so we can write it in the form $v g$, where $v \in C(X),|v|=1$ and $g \in A^{-1}$. [This is possible because $A$ is strongly logmodular]. Both the functions $v$ and $\bar{v}$ are in $A_{s}$. By Theorem $\mathrm{A}$ there exists $h$ in $A$ and an inner function $b$ which is invertible in $A_{S}$ such that $\|v-h \bar{b}\|_{\infty}<1$. Since $\bar{v} \bar{b} \in A_{S}$ and $\|1-\bar{v} \bar{b} h\|_{\infty}<1$, then by [2, p. 49] we have $\bar{v} \bar{b} h=e^{u_{1}}$ for some $u_{1}$ in $A_{s}$. By the definition of $A_{s}$, there exists $u$ in $A$ such that $u=u_{1}$ on $s$. Thus $v=\bar{b} h e^{-u}$ on $s$. By Theorem 3.2 there exists $F=\bar{b}$ on $s$. Set $G=F h e^{-u} g$, then $G$ is the required function. This completes the proof of the corollary.

ACKNowledgment. I would like to thank D. Luecking for a helpful discussion, and referee for helpful comments.

\section{REFERENCES}

1. S. Axler, Subalgebras of $L^{\infty}$, Thesis, University of California at Berkeley, 1975.

2. A. Browder, Introduction to Function Algebras, W. A. Ben jamin, Inc., N. Y., 1969.

3. K. Clancey and J. Gosselin, On the local theory of Toeplitz operators, Illinois J. Math., 22 (1978), 449-458.

4. T. W. Gamelin, Uniform Algebras, Prentice-Hall series in modern analysis, Prentice Hall, Inc., N. Y., 1969. 
5. K. Hoffman, Analytic functions and logmodular algebras, Acta Math., 108 (1962), 271-317.

6. K. Hoffman and H. Rossi, Extensions of positive weak*-continuous functionals, Duke Math. J., (1967), 34 453-466.

7. R. Younis, Extension results in the Hardy space associated with a logmodular algebra, J. Functional analysis, 39 (1980), 16-22.

Received May 5, 1980.

The University of Wisconsin-Milwaukee

Milwaukee, WI 53201 



\section{PACIFIC JOURNAL OF MATHEMATICS}

\section{EDITORS}

DONALD BABBITT (Managing Editor)

University of California

Los Angeles, California 90024

HUGO ROSSI

University of Utah

Salt Lake City, UT 84112

C. C. MOORE and ARTHur AGUS

University of California

Berkeley, CA 94720
J. DUGUNDJI

Department of Mathematics

University of Southern California

Los Angeles, California 90007

R. FINN and J. MILGRAM

Stanford University

Stanford, California 94305

\section{ASSOCIATE EDITORS}
R. Arnes
E. F. BECKENBACH
B. H. NeumanN
F. WOLF
K. YösHIDA

\section{SUPPORTING INSTITUTIONS}

UNIVERSITY OF ARIZONA

UNIVERSITY OF BRITISH COLUMBIA

CALIFORNIA INSTITUTE OF TECHNOLOGY

UNIVERSITY OF CALIFORNIA

MONTANA STATE UNIVERSITY

UNIVERSITY OF NEVADA, RENO

NEW MEXICO STATE UNIVERSITY

OREGON STATE UNIVERSITY
UNIVERSITY OF OREGON

UNIVERSITY OF SOUTHERN CALIFORNIA

STANFORD UNIVERSITY

UNIVERSITY OF HAWAII

UNIVERSITY OF TOKYO

UNIVERSITY OF UTAH

WASHINGTON STATE UNIVERSITY

UNIVERSITY OF WASHINGTON 


\section{Pacific Journal of Mathematics}

Vol. 102, No. $1 \quad$ January, 1982

S. Agou, Degré minimum des polynômes $f\left(\sum_{i=0}^{m} a_{i} X^{p^{r i}}\right)$ sur les corps finis

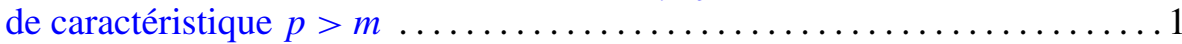

Chi Cheng Chen, On the image of the generalized Gauss map of a complete

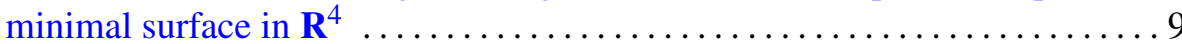

Thomas Curtis Craven and George Leslie Csordas, On the number of real

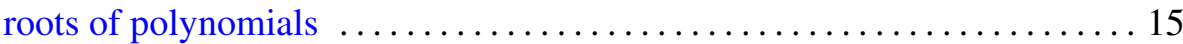

Allan L. Edelson and Kurt Kreith, Nonlinear relationships between oscillation and asymptotic behavior ....................... 29

B. Felzenszwalb and Antonio Giambruno, A commutativity theorem for

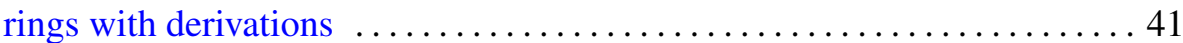

Richard Elam Heisey, Manifolds modelled on the direct limit of lines . . . . . 47

Steve J. Kaplan, Twisting to algebraically slice knots $\ldots \ldots \ldots \ldots \ldots \ldots 5$

Jeffrey C. Lagarias, Best simultaneous Diophantine approximations. II.

Behavior of consecutive best approximations $\ldots \ldots \ldots \ldots \ldots \ldots \ldots 61$

Masahiko Miyamoto, An affirmative answer to Glauberman's conjecture . . . 889

Thomas Bourque Muenzenberger, Raymond Earl Smithson and L. E.

Ward, Characterizations of arboroids and dendritic spaces ........... 107

William Leslie Pardon, The exact sequence of a localization for Witt

groups. II. Numerical invariants of odd-dimensional surgery

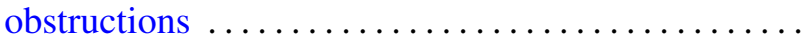

Bruce Eli Sagan, Bijective proofs of certain vector partition identities

Kichi-Suke Saito, Automorphisms and nonselfadjoint crossed products ...

John Joseph Sarraille, Module finiteness of low-dimensional PI rings ...

Gary Roy Spoar, Differentiable curves of cyclic order four . .

William Charles Waterhouse, Automorphisms of quotients of $\Pi \mathrm{GL}\left(n_{i}\right)$

Leslie Wilson, Mapgerms infinitely determined with respect to right-left equivalence

Rahman Mahmoud Younis, Interpolation in strongly logmodular

algebras 